\title{
Explorando Afinidade de Memória em Arquiteturas NUMA
}

\author{
Christiane Pousa Ribeiro \\ Vania Marangozova-Martin \\ Jean-François Méhaut \\ Laboratoire d'Informatique Grenoble - Grenoble - França \\ (Christiane.Pousa, Vania.Marangozova-Martin, Jean-Francois.Mehaut)@imag.fr \\ Fabrice Dupros \\ BRGM - Orléans, França \\ f.dupros@brgm.fr
}

\begin{abstract}
Resumo
Arquiteturas NUMA possuem latência e largura de banda assimétricas devido a existência de múltiplos níveis hierárquicos de memória no sistema. Para garantir desempenho neste tipo de arquitetura torna-se necessário garantir a afinidade de memória nas aplicações. Os sistemas operacionais, com suporte para arquiteturas NUMA, possuem políticas para alocação e escalonamento de memória e threads que visam a afinidade de memória. Entretanto, essas políticas não apresentam sempre o melhor desempenho para todos os tipos de aplicações. Ferramentas e APIs, presentes nestes sistemas operacionais, permitem gerenciar explicitamente a afinidade de memória nas aplicações. Neste trabalho será apresentado a avaliação de desempenho de diferentes estratégias para gerenciamento explícito de afinidade de memória, implementadas com APIs do sistema operacional em aplicações paralelas. Essas estratégias foram implementadas em uma aplicação sísmica e em kernels do Benchmark NAS e executadas em diferentes arquiteturas NUMA. Os resultados mostram a importância de garantir a afinidade de memória em arquiteturas NUMA (ganho médio de até $80 \%$ ) e que isso pode ser obtido através de APIs do sistema operacional.
\end{abstract}

\section{Introdução}

Arquiteturas NUMA (Non Uniform Memory Access) estão se tornando arquiteturas comuns em processamento paralelo. Isto pode ser explicado pelo surgimento das novas gerações de arquiteturas multicore, SMPs (Symetric Multiprocessor) e processadores multithread com múltiplos níveis de memória. Essas novas gerações são exemplos reais de arquitetura NUMA e têm sido amplamente utilizadas como máquinas para computação intensiva de aplicações paralelas [1]. A sua principal característica é a presença de vários níveis de hierarquia de memória distribuída compartilhados pelos elementos de processamento. Esta característica permite o desenvolvimento de aplicações usando o modelo de programação de memória compartilhada $[2,3]$. O principal problema nesse tipo de arquitetura é o tempo de acesso aos dados compartilhados. Como os elementos de processamento compartilham a memória distribuída, o tempo de acesso aos dados locais e remotos não são uniformes e isso pode diminuir o desempenho da aplicação $[2,4,5]$.

O desempenho das aplicações paralelas nessas arquiteturas é importante, uma vez que elas estão sendo usadas como máquinas para aplicações que demandam muito processamento e um baixo tempo de execução. Para otimizar o desempenho nas arquiteturas NUMA torna-se necessário minimizar o número de acessos remotos realizados pelas unidades de processamento, garantindo a afinidade de memória. A afinidade de memória está relacionada com a redução da distância entre os processos e dados usados por eles, ou seja, minimização do número de acessos remotos aos dados $[2,4,6]$.

Para garantir a afinidade de memória e assim aumentar o desempenho da aplicação, o sistema operacional provê políticas para gerenciar alocação de memória e escalonamento dos processos nas arquiteturas NUMA. Entretanto, estas políticas não apresentam sempre o desempenho ótimo para todos os tipos de aplicação. Então, nos últimos anos, os sistemas operacionais (Linux, Solaris, etc) têm provido ferramentas em nível de usuário e APIs com chamadas de sistemas que permitem aos programadores gerenciar explicita- 
mente a distribuição/alocação de memória e processos das suas aplicações, garantindo a afinidade de memória [5, 7].

Neste trabalho será apresentado a avaliação de desempenho de diferentes estratégias, implementadas com APIs do sistema operacional, que têm como objetivo garantir a afinidade de memória. Essas estratégias foram implementadas usando gerenciamento explícito de memória através de chamadas de sistema no código fonte das aplicações. As aplicações consideradas são kernels do benchmark NAS [8] e uma aplicação real que realiza simulação de sismos (Ondes 3D) [9]. Essas aplicações foram desenvolvidas em C e usando diretivas OpenMP [10] para o paralelismo do código. O principal objetivo deste trabalho é mostrar a possibilidade de obter-se ganhos importantes com gerenciamento explícito da afinidade de memória usando APIs do sistema operacional.

O restante do artigo está organizado da seguinte forma: Seção 2 apresenta os principais conceitos sobre arquiteturas NUMA. A Seção 3 discute os principais trabalhos relacionados. A seção 4 apresenta o método experimental, a aplicação sísmica, os kernels do benchmark NAS, as estratégias para gerenciamento de memória implementadas e as características das arquiteturas NUMA utilizadas. A Seção 5 apresenta os resultados obtidos e a Seção 6 as conclusões e possíveis trabalhos futuros.

\section{Arquiteturas NUMA}

Uma arquitetura NUMA pode ser definida como um conjunto de nodos que compartilham diferentes níveis de memória (caches e memória principal). Nessa arquitetura os tempos de acessos à memória não são uniformes. A assimetria no tempo de acesso acontece porque a memória global está fisicamente distribuída entre os nodos que compõem a arquitetura. Como esses nodos estão conectados através de uma rede, observamos o aparecimento do que chamamos de fator NUMA. O fator NUMA é a razão entre o tempo de acesso a um dado remoto (fisicamente alocado em outro nodo) e o tempo de acesso a um dado local (fisicamente alocado no bloco de memória presente no nodo). Nessas arquiteturas NUMA torna-se importante reduzir o impacto do fator NUMA sobre a aplicação [5, 3].

A redução do impacto do fator NUMA pode ser feita considerando dois tipos de otimizações: largura de banda e latência. Otimizar a largura de banda da rede de interconexão evita problemas de contenção de memória, permitindo muitos acessos concorrentes ao mesmo bloco de memória. A otimização da latênca evita grande número de acessos remotos, threads e dados são alocados próximos.

Além do fator NUMA, características como cache e falso compartilhamento devem ser consideradas quando desenvolve-se aplicações para essas arquiteturas. Nas arquiteturas NUMA deve-se otimizar o uso de memória cache e favorecer o princípio da localidade nas aplicações: maior número de acessos à memória cache significa menos acessos à memória principal, que no caso das arquiteturas NUMA pode ser local ou remota. Muitas vezes, nessas arquiteturas o desempenho pode ser prejudicado pelo falso compartilhamento. No falso compartilhamento processos/threads acessam dados que estão fisicamente localizados em uma mesma página de memória. Então, a página pode ser bloqueada por qualquer processo/thread que use um dos dados contidos nela, não permitindo que outro processo/thread tenha acesso aos outros dados contidos na página.

\subsection{Modelo de Programação}

Uma das principais vantagens de se utilizar arquiteturas NUMA é a possibilidade de desenvolver aplicações usando o modelo de programação de memória compartilhada. Nesse modelo de programação, as aplicações paralelas são desenvolvidas considerando que a memória é acessada por múltiplos fluxos de execução. Para desenvolver as aplicações considerando esse modelo o desenvolvedor pode utilizar APIs do sistema operacional ou de linguagens de programação. Como exemplos de APIs podemos citar, POSIX threads [11] e OpenMP [10].

OpenMP é um padrão muito utilizado para o desenvolvimento de aplicações paralelas no modelo de programação de memória compartilhada. As implementações deste padrão permitem, através de diretivas, paralelizar o código da aplicação de maneira simples e com um bom desempenho. Entretanto, como OpenMP foi proposto para arquiteturas UMA (Uniform Memory Access), não existe nenhum suporte para arquiteturas NUMA [10, 12]. Ou seja, nesse padrão não existe nenhuma otimização de memória para arquiteturas com múltiplos níveis de memória compartilhada.

\subsection{Suporte do Sistema Operacional}

O suporte para gerenciamento de memória em arquiteturas NUMA está atualmente presente em grande parte dos sistemas operacionais. Esse suporte pode ser tanto no nível de usuário (através de ferramentas/comandos) quanto no nível de chamadas de sistema (através de APIs). Podemos citar como exemplos desses sistemas operacionais, o Linux e o Solaris.

O suporte no nível de usuário usando ferramentas ou comandos permite ao desenvolvedor da aplicação especificar a política de memória e de escalonamento de threads para uma aplicação. A vantagem de usar esse tipo de suporte é que nenhuma modificação no código fonte da aplicação é necessário. Entretanto, essa solução afeta a aplicação 
como um todo, não é permitido alterar as políticas durante a execução da aplicação [5].

A API NUMA é uma interface que define um conjunto de chamadas de sistema que afetam a alocação de memória e threads. Neste caso, o desenvolvedor deve ter acesso ao código fonte da aplicação para gerenciar a memória e a alocação de threads. A principal vantagem de usar APIs é a possibilidade de um controle mais fino da alocação de memória/threads [5, 13].

\section{Trabalhos Relacionados}

Em [7], os autores apresentam diferentes estratégias para alocação de memória e processos/threads em duas plataformas diferentes (UltraSparc-Solaris e Opteron-Linux) em nível de usuário. As estratégias usam ferramentas do sistema operacional para alocação e não APIs de chamadas do sistema. Além disso, os autores fazem uma avaliação da latência e largura de banda das duas plataformas e propõem um framework para realizar experimentos de alocação de memória e processos/threads nas duas plataformas. Os autores mostram em seus resultados que o uso do framework proposto pode ajudar os desenvolvedores a otimizar a afinidade de memória nas suas aplicações.

No trabalho [14] é apresentado a avaliação do impacto da migração de páginas de memória no sistema. Os autores avaliaram diferentes aplicações OpenMP (disponibilizadas nos benchmarks NAS e SPEC) em diferentes condições: política de escalonamento e carga do sistema. Os resultados mostram que ganhos de desempenho podem ser obtidos com o uso de migração de páginas se a política de escalonamento considera as características de memória.

Em [15], os autores apresentam um estudo sobre o desempenho alcançado por aplicações reais que usam o suporte do sistema operacional para migrar/replicar os dados. Com o suporte oferecido pelo sistema operacional os autores minimizam os efeitos NUMA sobre as aplicações e apresentam ganhos de até $30 \%$.

No trabalho [3], os autores apresentam uma nova estratégia de alocação de memória, on-next-touch. Essa estratégia permite que os dados sejam migrados para os nodos onde as threads que os utilizam estão sendo executadas. A avaliação desta nova estratégia é feita considerando uma aplicação real que possui diferentes padrões de acesso. Os autores apresentam ganhos de até $69 \%$ com 22 threads.

Os trabalhos apresentados não consideram uma avaliação de desempenho de diferentes aplicações reais e benchmarks com padrões de acessos regulares e irregulares aos dados. Além disso, os trabalhos não avaliaram aplicações desenvolvidas com OpenMP e chamadas de sistema que visam minimizar o impacto dos efeitos NUMA e garantir a afinidade de memória.

\section{Avaliação de Estratégias para Afinidade de Memória}

Nesta seção é apresentado o método experimental, as diferentes estratégias implementadas, as aplicações e as plataformas NUMA utilizadas.

Os experimentos foram realizados considerando duas arquiteturas NUMA e diferentes implementações das aplicações. A métrica selecionada para avaliação de desempenho foi o tempo de execução da aplicação, que é uma das métricas mais utilizadas e representativas dentro de processamento paralelo.

\subsection{Chamadas de Sistema}

Neste trabalho, as estratégias para otimizar a afinidade de memória foram desenvolvidas utilizando o suporte do sistema operacional Linux. A escolha do Linux foi principalmente por ele ser um dos sistemas operacionais mais utilizados em arquiteturas NUMA.

Desde a versão 2.6 do kernel o Linux oferece suporte para afinidade de memória em NUMA $[5,7]$. A política padrão de alocação de memória usada no Linux é a FirstTouch, que consiste em alocar o dado no bloco de memória do nodo que fez o acesso primeiro. Em máquinas NUMA a política padrão do Linux para alocar as threads tem como objetivo minimizar a distância entre as threads e os dados $[16,5]$.

Aplicações paralelas OpenMP são geralmente desenvolvidas usando uma de duas estratégias de gerenciamento de afinidade de memória:

- First-Touch: diretivas OpenMP são usadas apenas para paralelizar as etapas de cálculo da aplicação. Não existe otimização para aspectos de memória (alocação física dos dados) e a política padrão do Linux (Firsttouch) é usada para alocação dos dados. Ou seja, thread mestre aloca e inicializa todas as estruturas de dados, mantendo-as em um único nodo da máquina NUMA.

- Parallel-Init: nesta versão são adicionadas diretivas OpenMP na etapa de inicialização das estruturas de dados. Desta forma, a inicialização das estruturas de dados também é realizada de forma paralela e dados são alocados fisicamente no mesmo nodo da thread que os utiliza. Essa solução é amplamente utilizada pelos desenvolvedores de aplicações paralelas OpenMP. Entretanto, ela apresenta bons resultados apenas quando o padrão de acesso aos dados é regular e a carga de trabalho de cada thread é definida estaticamente. 
O desenvolvimento de aplicações paralelas usando OpenMP pode ser realizado como apresentado acima. Entretanto, não existe nenhuma garantia de afinidade de memória e o melhor desempenho provavelmente não será alcançado.

Em arquiteturas NUMA, a afinidade de memória pode ser garantida através do controle explícito da alocação de memória e threads da aplicação. Esse controle pode ser obtido através do uso da APIs do sistema operacional que permitem um controle fino da alocação de memória e threads na aplicação.

Este trabalho, foca-se na API NUMA de chamadas de sistema e as duas funções usadas para garantir a afinidade de memória são: mbind e sched_setaffinity [17, 13]. Estas duas funções permitem que o programador especifique a política de memória para uma seqüência de dados e onde os processos/threads serão executados. Então, o programador pode minimizar os efeitos NUMA em uma aplicação na arquitetura NUMA escolhida.

A função mbind usa alguns parâmetros para especificar a política de memória a ser adotada, os nodos que serão usados para alocação, a sequência de páginas e um flag que permite realizar a migração de páginas. As políticas de memória permitidas para o mbind são: bind (memória será apenas alocada em um conjunto pré-definido de nodos), interleave (memória será alocada ciclicamente em um conjunto de nodos, o nodo inicial é aquele que primeiro causou a falta de página) e preferred (mémoria será preferencialmente alocada em um conjunto espedífico de nodos) [18]. A função sched_setaffinity utiliza três parâmetros: o identificador do processo/thread, uma máscara que indica o processador/núcleo a ser utilizado e um último parâmetro que informa o tamanho da máscara [17].

Abaixo apresentamos duas possíveis estratégias de afinidade de memória que usam as funções citadas.

- Round-Robin: todos os dados da aplicação são alocados fisicamente na máquina usando a política de memória interleave da chamada de sistema mbind. As estruturas de dados são alocadas dinamicamente usando a chamada de sistema mmap que retorna um ponteiro para a primeira página de memória que contém os dados da estrutura. Usando esse ponteiro na função mbind informamos a política de memória que deve ser usada nesse conjunto de páginas. A implementação com interleave, favorece a largura de banda pois os dados são distribuídos ciclicamente nos nodos utilizados durante a execução da aplicação. Essa estratégia minimiza o número de acessos concorrentes no mesmo bloco de memória. As threads são alocadas nos processadores/núcleos usando a chamada de sistema sched_setaffinity e considerando o seu identificador OpenMP como número do processador/núcleo. A alocação das threads permite contro- lar as migrações realizadas pelo sistema operacional garantindo a afinidade de memória.

- Memory-Bind: utiliza chamadas de sistema para alocar dados e threads na arquitetura NUMA. As funções usadas são: mbind e sched_setaffinity. Considerando a função mbind e a política bind, apenas os dados mais importantes da aplicação (maior consumo de memória e número de acessos) são alocados com esta política. As estruturas de dados nessa implementação também são alocadas dinamicamente e o ponteiro retornado é usado para definir a política de memória para a seqüencia de páginas. Na implementação com bind, a latência é favorecida pois, os dados são alocados no mesmo nodo que a thread que vai utilizá-los. As threads são alocadas nos processadores/núcleos considerando os seus identificadores OpenMP como número do processador/núcleo, isso facilita o controle da sequiência de dados que deve ser alocada no nodo.

Essas estratégias foram empregadas na implementação das aplicações apresentadas na seção 4.2.

\subsection{Aplicações}

\subsubsection{Ondes 3D}

A aplicação Ondes 3D é uma aplicação real que simula a propagação de ondas em uma região. Esta aplicação é utilizada na previsão de sismos e geotérmicos. A sua principal característica é o alto consumo de memória (grandes matrizes de três dimensões) e de processamento. A aplicação possui três grandes etapas: a primeira é responsável pela inicialização das estruturas de dados, a segunda por calcular a velocidade da propagação da onda e a terceira pelo cálculo do estresse na região. O padrão de acessos aos dados durante as três etapas da aplicação é regular (threads sempre acessam o mesmo conjunto de páginas). A aplicação foi desenvolvida em $\mathrm{C}$ utilizando diretivas OpenMP para a paralelização do código [9].

\subsubsection{Benchmark NAS}

O Benchmark NAS é um conjunto de aplicações derivadas de aplicações espaciais (dinâmica de fluídos) usado amplamente para avaliação de desempenho em processamento paralelo. Esse benchmark é composto de cinco kernels e três aplicações que simulam a dinâmica de fluídos [8]. A principal diferença entre um kernel e uma aplicação é que o primeiro representa o núcleo de computação de métodos numéricos e a segunda simula a movimentação de dados e computação que existe em dinâmica de fluídos.

Para os experimentos foram selecionados os kernels $\mathrm{CG}$ e MG por eles terem como principais características um 
padrão de acesso irregular aos dados (threads não trabalham sempre com o mesmo conjunto de páginas da memória), grandes conjuntos de estruturas de dados (alto consumo de memória) e grande número de acesso a eles. O CG é um kernel que utiliza o método Conjugate Gradient para calcular o menor auto-valor em uma matriz de grande dimensão e não estruturada. O kernel MG utiliza o método $V$-cycle MultiGrid para calcular a solução 3D para equação de Poisson.

\subsection{Plataforma Experimental}

Nos experimentos foram utilizados duas máquinas NUMA diferentes. A primeira, é um Itanium 2 composta por 16 processadores de 1,6 GHz de freqüência e memória principal total de 64 Gbytes. A máquina está organizada em quatro nodos com quatro processadores com cache L3 compartilhada de 9 Mbytes. Esta memória principal está dividida em 4 blocos de 16 Gbytes, um em cada nodo da arquitetura. A largura de banda para acesso à memória principal é de 1.157,05 Mbytes/sec para leituras e 780,33 Mbytes/sec para escritas (resultados obtidos com Stream Benchmark [19]). Os nodos são conectados usando FAME Scalability Switch (FSS) que é um backplane proprietário desenvolvido pela BULL (www.bull.fr). Esta conexão gera diferentes latências na arquitetura, fator NUMA de 2 a 2,5. A distribuição Linux usada nesta máquina é a Bull Linux AS4 V5.1 (versão Kernel 2.6.18-intel-64). Os compiladores usados são: Intel C Compiler (ICC) e GNU Compiler Collection (GCC). A Figura 1 apresenta o esquema desta arquitetura.

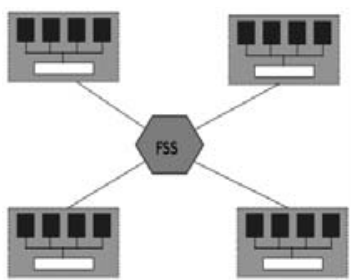

Figura 1. Arquitetura NUMA - Itanium2

A segunda arquitetura é um AMD Opteron de 8 processadores dual core com 2,2 GHz e cache L2 de 2 Mbytes para cada processador. A máquina está organizada em 8 nodos, uma capacidade total de 32 Gbytes de memória, dividida igualmente entre os nodos. A largura de banda para acesso a memória principal é de 1.617,08 Mbytes/sec para leituras e 1.458,89 Mbytes/sec para escritas (resultados obtidos com Stream Benchmark [19]). Cada nodo possui três conexões que são usadas para conectar com outros nodos ou com cartões de entrada/saída. Essas conexões geram diferentes latências na arquitetura, fator NUMA de 1,2 a
1,5. A distribuição Linux usada nesta máquina é a Debian (versão Kernel 2.6.23-1amd64). Os compiladores usados são: Portland Group Inc. (PGI) e GCC. A Figura 2 apresenta o seu esquema.

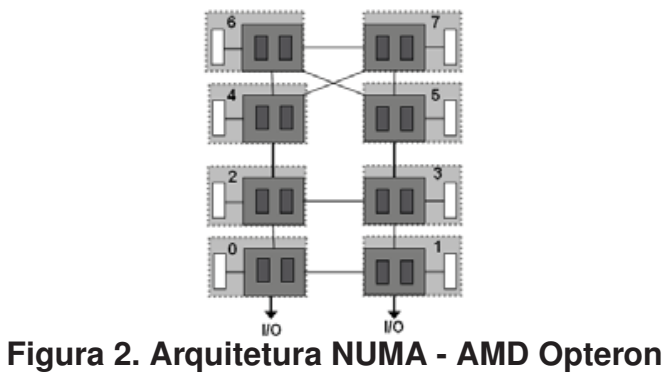

\section{Resultados}

Esta seção apresenta a avaliação de desempenho das diferentes implementações das aplicações (First-Touch, Parallel-Init, Round-Robin e Memory-Bind) nas duas arquiteturas selecionadas. Os testes foram realizados com 2, 4, 8 e 16 threads e instância do problema de tamanho 2,4 Gbytes para aplicação real Ondes 3D e classe A ( $256^{2}$ elementos e 20 iterações) para as aplicações do benchmark NAS. Os tempos de execução apresentados, para cada implementação e número de threads, são valores médios com validade estatística.

\subsection{Arquitetura Itanium}

A Figura 3 apresenta o tempo de execução obtido com a aplicação Ondes 3D (Compilador ICC) quando executada na arquitetura Itanium. Como podemos observar os resultados obtidos com a estratégia Memory-Bind foram na média $22 \%$ melhores que os resultados obtidos com a solução Round-Robin. Essa máquina possui fator NUMA alto, dados e threads devem ser alocados considerando uma distância mínima entre eles quando a aplicação tem como principal característica um padrão de acessos regular aos dados. Nesse caso, distribuir os dados ciclicamente gera um número maior de acessos remotos. Considerando a implementação First-touch, os resultados obtidos foram 5\% piores que os resultados obtidos com Memory-Bind. Nesta implementação os dados são alocados fisicamente no nodo do processo mestre e as threads fazem acessos remotos e concorrentes para acessálos.

Outro resultado interessante é que os resultados obtidos com a implementação Memory-Bind são próximos aos resultados obtidos com Parallel-Init. Como a implementação Parallel-Init usa diretivas OpenMP 


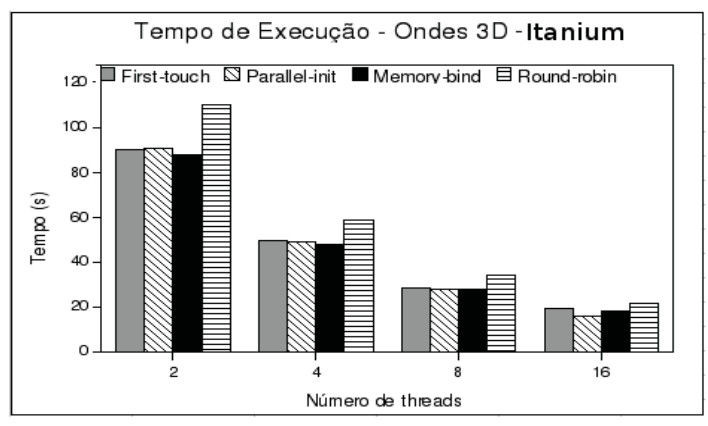

Figura 3. Tempo Execução - Ondes 3D - Itanium

na etapa de inicialização e a aplicação tem padrão de acesso regular, os dados são alocados fisicamente próximos das threads que os utilizam. Entretanto, é importante ressaltar que os resultados obtidos com Memory-Bind foram 2\% melhores que os obtidos com Parallel-Init.

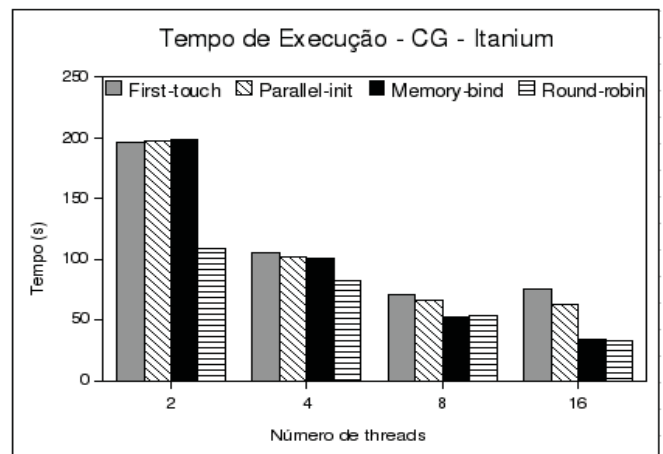

Figura 4. Tempo Execução - CG - Itanium

A Figura 4 fornece o tempo de execução obtido com o kernel CG (Compilador ICC) quando executado na arquitetura Itanium. Os resultados obtidos com Round-Robin foram na média $80 \%$ melhores que a versão First-Touch e 50\% melhores que Parallel-Init. O kernel possui padrão de acesso irregular aos dados e distribuir as páginas de memória que contêm os dados ciclicamente entre os nodos da máquina NUMA, maximiza o número de acessos locais. A estratégia Memory-Bind apresentou resultados melhores que as estratégias First-Touch e Parallel-Init (27\% e 39\%), porém não melhores que a estratégia Round-Robin. Essa estratégia realiza muitas migrações de páginas da memória para minimizar o número de acessos remotos. Entretanto, o custo das migrações é alto pois, o padrão de acesso aos dados muda muito ao longo da aplicação (threads acessam diferentes conjuntos de páginas ao longo da execucão).

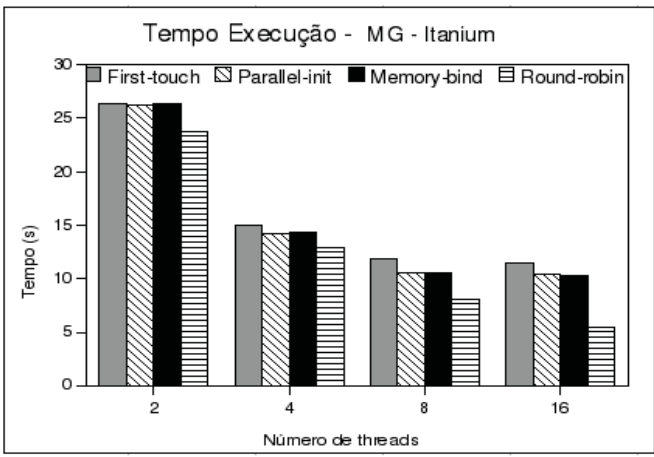

Figura 5. Tempo Execução - MG - Itanium

A Figura 5 mostra o tempo de execução obtido com o kernel MG (Compilador ICC) quando executado na arquitetura Itanium. Pode-se observar que a estratégia Round-Robin, na média, apresenta tempos de execução 45\% melhores que First-Touch e 35\% melhores que Parallel-Init. Assim, como o kernel CG, este kernel possui grandes estruturas de dados com padrão de acesso irregular. Então, distribuir os dados ciclicamente entre os nodos da máquina minimiza o número de acessos remotos e o número de migrações de páginas da memória. Como a máquina apresenta um custo alto para acessos remotos, minimizar o número de acessos remotos produz o melhor desempenho. Outro resultado importante, é a proximidade dos tempos de execução obtidos com as estratégias Parallel-Init e Memory-Bind. Nesse caso, o custo das migrações das páginas memória torna-se comparável ao custos dos acessos remotos que as threads realizam com a estratégia Parallel-Init.

A arquitetura Itanium possui um fator NUMA alto e uma largura de banda para memória local pequena. Portanto, neste tipo de arquitetura torna-se interessante trabalhar na latência para minimizar o número de acessos remotos e migração de páginas da memória.

\subsection{Arquitetura Opteron}

A Figura 6 mostra o tempo de execução obtido com a aplicação real Ondes 3D (Compilador PGI) executada na arquitetura Opteron. Como podemos observar, os resultados obtidos com a estratégia Round-Roubin foram, na média, $7 \%$ melhores que os resultados obtidos com a solução Parallel-Init. Essa máquina possui fator NUMA baixo, ou seja, a latência para acessos remotos é baixa. Portanto, a melhor solução é distribuir os dados entre os nodos minimizando o número de acessos concorrentes ao mesmo bloco de memória (contenção de memória).

Considerando ainda a Figura 6, observa-se que os piores 


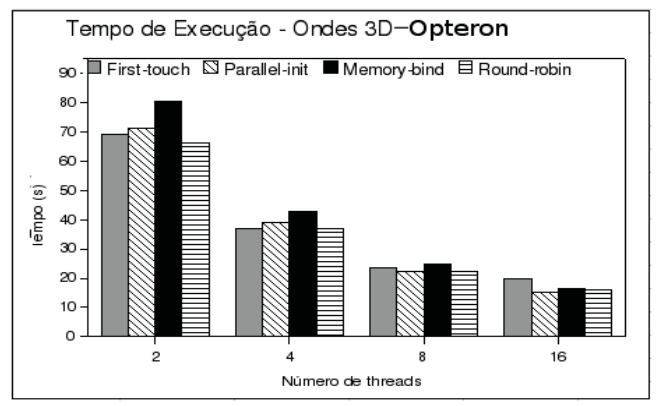

Figura 6. Tempo Execução - Ondes 3D Opteron

resultados foram obtidos com a estratégia Memory-Bind. Nesta estratégia apenas as estruturas mais importantes foram usadas na alocação explícita com mbind. Na versão Round-Robin, a política interleave foi usada em toda a memória de dados da aplicação, ou seja, afinidade de memória garantida para todos os dados.

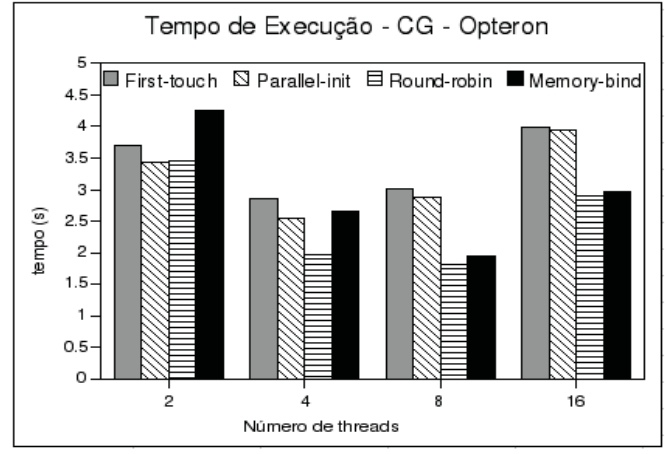

Figura 7. Tempo Execução - CG - Opteron

A Figura 7 apresenta o tempo de execução obtido com o kernel GC do NAS quando executado na arquitetura Opteron. Os resultados obtidos com a estratégia Round-Roubin foram, na média, 30\% melhores que os resultados obtidos com as outras soluções. Essa máquina possui um fator NUMA pequeno e priorizar a largura de banda à latência gera melhores resultados. Além disso,este kernel possui como principal característica acessos irregulares à matriz. Então, distribuir os dados ciclicamente entre os nodos da máquina, como na estratégia Round-Roub in, favorece a largura de banda e o padrão de acesso.

Outro resultado importante é a degradação do desempenho (escalabilidade) com o aumento do número de threads utilizadas. Essa degradação esta principalmente ligada aos aspectos de cache e a forma como a aplicação foi paralelizada com as diretivas OpenMP. A paralelização do código foi realizada considerando laços de grão fino e um número maior de threads gera uma sobrecarga maior nestes laços. O custo de criar/lançar as threads é grande se comparado ao trabalho a ser realizado.

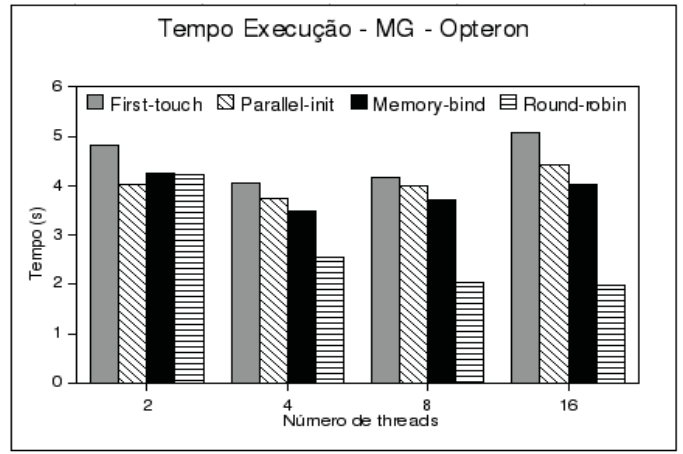

Figura 8. Tempo Execução - MG - Opteron

Por fim, a Figura 8 ilustra o tempo de execução obtido com o kernel MG (compilada com GCC) executado na arquitetura Opteron. Os resultados apresentados nessa figura, assim como os das duas figuras anteriores, mostram que os melhores tempos de execução são obtidos com a estratégia Round-Roubin. Esta solução favorece a largura de banda e, conseqüentemente, gera os melhores resultados. Considerando os resultados obtidos com a estratégia Memory-Bind, pode-se observar que, na média, são melhores que os resultados da estratégia Parallel-Init. $\mathrm{Na}$ estratégia Memory-Bind são realizadas migrações de páginas quando as threads acessam outro conjunto de dados.

Ainda na Figura 8, podemos observar que a solução Round-Roubin é a única que não apresenta degradação da aceleração (speedup). As demais soluções apresentam diminuição no ganho obtido quando aumentamos o número de threads. Esse resultado pode ser explicado pelas características da máquina e da aplicação. Esta máquina NUMA possui problema de contenção, logo, alocar as páginas considerando quem primeiro fez acesso a elas (First-Touch e Parallel-Init) aumenta o número de acessos concorrentes ao mesmo bloco de memória. Além disso, a aplicação possui padrão de acessos irregular; threads não acessam sempre o mesmo conjunto de dados.

A arquitetura Opteron possui um fator NUMA pequeno que torna o custo dos acessos remotos próximos ao custo dos acessos locais. Além disso, a largura de banda da memória local é alta, ou seja, tempo de acesso à memória local é comparável ao tempo de acesso a memória cache L2. Conseqüentemente, otimizar a largura de banda produz melhores resultados que otimizar a latência. Nossos resultados confirmam isso, pois a estratégia de afinidade de memória Round-Robin, que distribui threads e dados nos 
nodos da máquina NUMA produziu os melhores resultados para as aplicações estudadas.

\section{Conclusões}

Neste artigo, foi apresentado a avaliação de desempenho de diferentes estratégias para afinidade de memória em uma aplicação real e em kernels do benchmark NAS. Essas estratégias foram implementadas com chamadas de sistema do Linux e permitem um controle explícito da alocação de memória e threads em máquinas NUMA.

Os resultados apresentados mostram a importância de gerenciar a memória das aplicações que são executadas em máquinas NUMA. Observamos ganhos de até $80 \%$ com as estratégias que usam chamadas do sistema operacional para otimizar afinidade de memória. O trabalho realizado justifica a importância de escolher a estratégia a ser usada na aplicação e que esta, depende de características da máquina NUMA e da própria aplicação. As chamadas do sistema permitem ao desenvolvedor implementar aplicações considerando as suas características e as da arquitetura NUMA podendo gerar ganho médio entre $2 \%$ - $80 \%$ em relação às implementações que não consideram afinidade de memória.

A principal contribuição deste trabalho é a avaliação de desempenho de diferentes estratégias para afinidade de memória em arquiteturas NUMA implementadas com APIs do sistema operacional. Como trabalhos futuros nós destacamos: estudo de mecanismos para a gerência de memória em outros sistemas operacionais, identificação de características da arquitetura e da aplicação que influenciam a afinidade de memória e proposta de um framework para controle da afinidade de memória em arquiteturas NUMA.

\section{Agradecimentos}

Agradecemos o apoio financeiro dos órgãos e projetos NUMASIS, ANR, Bull e CAPES (Processo 4874-06-4) na realização deste trabalho.

\section{Referências}

[1] Top500 supercomputing sites - http://www.top500.org, 2008.

[2] T. Mu, J. Tao, M. Schulz, and S. A. Mckee. Interactive Locality Optimization on NUMA Architectures. In Software Visualization, 2003.

[3] H. Löf and S. Holmgren. Affinity-on-next-touch: Increasing the performance of an industrial PDE solver on a cc-NUMA system. In ICS '05: Proceedings of the 19th annual international conference on Supercomputing, pages 387-392, New York, NY, USA, 2005. ACM.

[4] J. Marathe and F. Mueller. Hardware Profile-Guided Automatic Page Placement for ccNUMA Systems. In PPoPP
'06: Proceedings of the eleventh ACM SIGPLAN symposium on Principles and practice of parallel programming, pages 90-99, New York, NY, USA, 2006. ACM.

[5] A. Carissimi, F. Dupros, J.-F. Mehaut, and R. V. Polanczyk. Aspectos de Programacão Paralela em arquiteturas NUMA. In VIII Workshop em Sistemas Computacionais de Alto Desempenho, 2007.

[6] F. Bellosa and M. Steckermeier. The Performance Implications of Locality Information Usage in Shared-Memory Multiprocessors. J. Parallel Distrib. Comput., 37(1):113121, August 1996.

[7] A. Joseph, J. Pete, and R. Alistair. Exploring Thread and Memory Placement on NUMA Architectures: Solaris and Linux, UltraSPARC/FirePlane and Opteron/HyperTransport. High Performance Computing HiPC 2006, pages 338-352, 2006.

[8] J. Y. Haoqiang Jin, Michael Frumkin. The OpenMP Implementation of NAS Parallel Benchmarks and Its Performance. Technical Report 99-011/1999, NAS System Division - NASA Ames Research Center, 1999.

[9] F. Dupros, H. Aochi, A. Ducellier, D. Komatitsch, and J. Roman. Exploiting intensive multithreading for the efficient simulation of seismic wave propagation. In 11th International Conference on Computational Science and Engineering, Sao Paulo, Brazil, July 2008.

[10] The openmp specification for parallel programming http://www.openmp.org, 2008.

[11] F. Garcia and J. Fernandez. Posix thread libraries. Linux J., page 36, 2000.

[12] C. Terboven, An, and S. Sarholz. Openmp on multicore architectures. In A Practical Programming Model for the Multi-Core Era, pages 54-64. Springer, 2008.

[13] A. Kleen. A NUMA API for LINUX. Technical report, Novell, April 2005.

[14] J. Corbalan, X. Martorell, and J. Labarta. Evaluation of the memory page migration influence in the system performance: the case of the SGI O2000. In ICS '03: Proceedings of the 17th annual international conference on Supercomputing, pages 121-129, New York, NY, USA, 2003. ACM.

[15] B. Verghese, S. Devine, A. Gupta, and M. Rosenblum. Operating system support for improving data locality on CCNUMA compute servers. In ASPLOS-VII: Proceedings of the seventh international conference on Architectural support for programming languages and operating systems, pages 279-289, New York, NY, USA, 1996. ACM.

[16] J. Bircsak, P. Craig, R. Crowell, Z. Cvetanovic, J. Harris, C. A. Nelson, and C. D. Offner. Extending OpenMP for NUMA machines. In Supercomputing '00: Proceedings of the 2000 ACM/IEEE conference on Supercomputing (CDROM), 2000.

[17] R. Love. Kernel korner: CPU affinity. Linux Journal, 2003(111):8, 2003.

[18] L. T. Schermerhorn. Automatic Page Migration for Linux. Linux, 2007.

[19] J. D. Mccalpin. STREAM: Sustainable memory bandwidth in high performance computers, 1995. 